\title{
Load balance recovery for multi-drop distribution problems: A mixed integer linear programming approach
}

\author{
Elsa Silva ${ }^{\mathrm{a}, *}$, António G. Ramos ${ }^{\mathrm{b}}$, José F. Oliveira ${ }^{\mathrm{c}}$ \\ a INESC TEC, Portugal \\ ${ }^{\mathrm{b}}$ INESC TEC and CIDEM, School of Engineering, Polytechnic of Porto, Portugal \\ ' INESC TEC, Faculty of Engineering, University of Porto, Portugal
}

\section{A R T I C L E I N F O}

\section{Article history:}

Received 12 June 2018

Revised 2 August 2018

Accepted 3 August 2018

Available online 11 August 2018

\section{Keywords:}

Container loading

Load balance

Multi-drop delivery

\begin{abstract}
A B S T R A C T
In road freight transport, a loaded vehicle with a distribution route and a compliant load balance at the depot can become non-compliant during the route, since the total weight of the cargo and its centre of gravity change with each delivery.

Nowadays, vehicles circulating on our roads either undermine safety regulations or lack operational efficiency when these regulations are taken into account and cargo is extensively rearranged after each delivery. This issue has been completely ignored both in the vehicle routing literature and in the container loading literature.

The aim of this work is to provide tools capable of ensuring that a cargo arrangement is load balanced along the complete distribution trip. It proposes a multi-drop load balance recovery algorithm (MDLBRA), which seeks to ensure that, when both a complete route and the respective cargo arrangement are provided, the boxes to be removed from the cargo arrangement at the depot and the boxes to be rearranged at each customer are identified, allowing the cargo to remain balanced after every delivery. It is important to notice that a MDLBRA is not a container loading algorithm: a MDLBRA modifies solutions generated by any container loading algorithm so that load balance is guaranteed when the truck leaves the depot and during the entire distribution route.
\end{abstract}

A mixed integer linear programming (MILP) model is proposed to balance the cargo at each customer stop. The MILP model incorporates load distribution diagram constraints in order to determine the feasible domain for the location of the centre of gravity of the cargo arrangement, taking into account the regulatory requirements and the technical characteristics of the vehicle.

Extensive computational experiments show that a MDLBRA can be used in practical contexts, as the MILP model was able to find a solution in less than ten minutes in $93 \%$ of the unbalanced test instances.

(C) 2018 Elsevier Ltd. All rights reserved.

\footnotetext{
* Corresponding author.

E-mail addresses: emsilva@inesctec.pt (E. Silva), agr@isep.ipp.pt (A.G. Ramos), jfo@fe.up.pt (J.F. Oliveira).
} 


\section{Introduction}

One of the main challenges of today's supply chains is to find ways of becoming economically, environmentally and socially efficient and sustainable, something that is getting more and more difficult due to the increasing volume of transported goods. Additionally, in Europe, Eurostat surveys estimate that a fifth of the trips of freight vehicles at the EU-28 level are performed by empty vehicles (Eurostat, 2017), i.e. the load factor of road freight transport is low. Altogether, this has led to high road congestion and $\mathrm{CO}_{2}$ emissions.

The optimisation of freight transport has been widely studied in literature, namely through the study of the capacitated vehicle routing problem (CVRP) and its variants. The CVRP tackles the distribution of goods and services between depots and customers, using a fleet of vehicles with limited capacity along a set of routes that aim to optimise one or more objectives. Its formulation is often extended by including additional constraints that are required in order to meet the needs of transport companies and customers. Examples include time windows in which a customer is available to receive a delivery, maximum duration of a route or controlling the truck speed to achieve a more sustainable distribution of goods.

Despite having a strong impact in the operational efficiency of the routes, loading constraints are mainly addressed in the container loading problem (CLP). In the CLP, a set of boxes must be packed inside a container in a way that maximises space utilisation while not overlapping said boxes. The problem is frequently extended by applying practical constraints such as weight limit, weight distribution, stability, multi-drop, load-bearing or load balance (Bortfeldt and Wäscher, 2013). All research hitherto has addressed these constraints with the container at its full load status.

Recent changes in EU regulation have placed a new focus on the load balance constraint. This constraint has been tackled within the CLP literature in an unrealistic way that considers the geometrical centre of the base of the container to be the ideal location for the centre of gravity of the cargo and that treats it mainly as a soft constraint, which in road transport does not comply with the corresponding legislation nor with vehicle specifications.

This scenario has only changed with the approach proposed by Ramos et al. (2018), which considers load balance a hard constraint and adopts vehicle specific load diagrams in order to define the feasibility domain for the location of the centre of gravity of the cargo, according to the technical characteristics of each vehicle and to real-world regulations and legislation.

Vehicle routing algorithms for road distribution that address multi-drop and cargo loading constraints still ignore the state of the vehicle with respect to load balance (Pollaris et al., 2015). For the sake of ensuring the efficiency of logistical operations, each stop at a customer should be as short as possible, therefore avoiding or minimising the cargo reallocation required to achieve a balanced cargo.

The centre of gravity of the cargo changes with each new delivery and in regard to load balance, the argument that a less than full vehicle will always comply with the transport regulation is not valid. The cargo balance has to be monitored not only at the point of departure but also throughout the entire trip. Currently, vehicles circulating on our roads either undermine safety regulations or lack operational efficiency when these regulations are taken into consideration and cargo is extensively rearranged after stopping at each customer.

In this work, the load balance of cargo arrangements generated for the container loading problem with multi-drop constraints is ensured throughout the entire trip, i.e. from the depot to the last customer. It proposes a mixed-integer linear program (MILP) that allows the recovery of load balance at the depot and at each customer stop, while minimising the number of boxes to move, as long as a complete route and the respective cargo arrangement are provided.

The remainder of the paper is organised as follows. The next section formally defines the problem. Section 3 presents a literature review and Section 4 demonstrates the MILP for load balance recovery. Section 5 shows the overall approach to load balance recovery for the multi-drop container loading problem. Section 6 contains extensive computational experiments with the multi-drop load balance recovery algorithm, and its underlying MILP model, when a route is provided. Section 7 presents computational experiments that analyse the impact of the multi-drop load balance recovery algorithm in container loading solutions under multi-drop constraints, this time generated under different route scenarios. Finally, Section 8 presents a summary of the results and the final conclusion.

\section{Problem definition}

The problem addressed in this work, named "multi-drop load balance recovery problem", can be exemplified as follows: given a cargo arrangement $P$ loaded in a cargo container $C$, which is to be transported by vehicle $V$ in an assigned distribution route with $m$ destinations (customers $k=1, \ldots, m), P$ should comply with regulatory and technical load balance requirements. However, $P$ may not meet these regulatory and technical load balance requirements both at the beginning of the route, at the depot, and along the route, since the total weight of the cargo and its centre of gravity change with each delivery to a customer. The aim is to decide which boxes must be removed from $P$ at the depot, in order to ensure that $P$ complies with load balance requirements, and which boxes must be moved and where to in $C$, after unloading cargo at a customer and before continuing the trip, ensuring cargo load balance along the complete route. The objective is to minimise the number of boxes removed from $P$ and moved after each delivery.

Taking the first octant of a Cartesian coordinates system, with the back-bottom-left corner of the container lying at the origin of the coordinates system, cargo arrangement $P$ is defined by a set of $n$ boxes $b_{i}(i=1, . ., n)$, each characterised by its depth $\left(d_{i}\right)$, width $\left(w_{i}\right)$, height $\left(h_{i}\right)$ (dimensions parallel to the $x, y$ and $z$ axes of the coordinates system) and weight (wgt $t_{i}$, loaded in a predetermined position, $\left(x_{i}^{\text {ini }}, y_{i}^{\text {ini }}, z_{i}^{\text {ini }}\right)$, inside container $C$. 


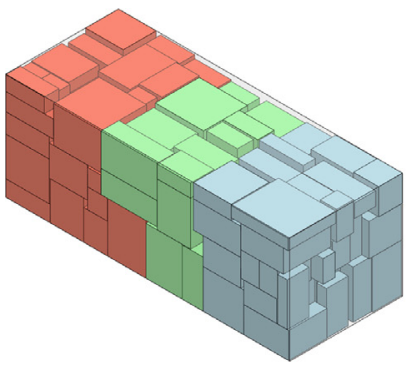

(a) Customers 1, 2 and 3

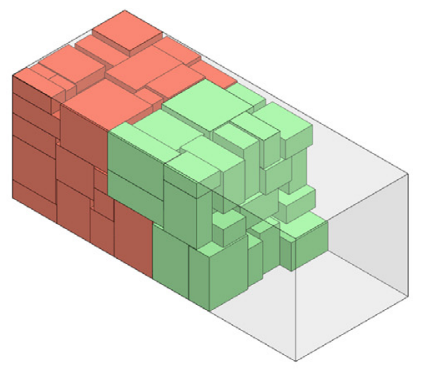

(b) Customers 2 and 3

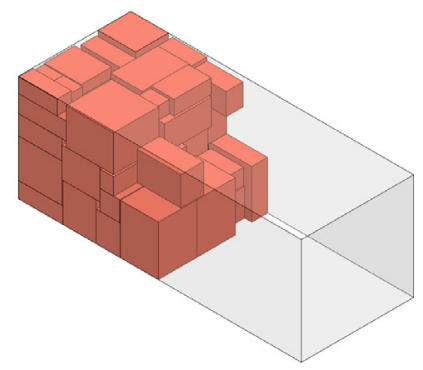

(c) Customer 3

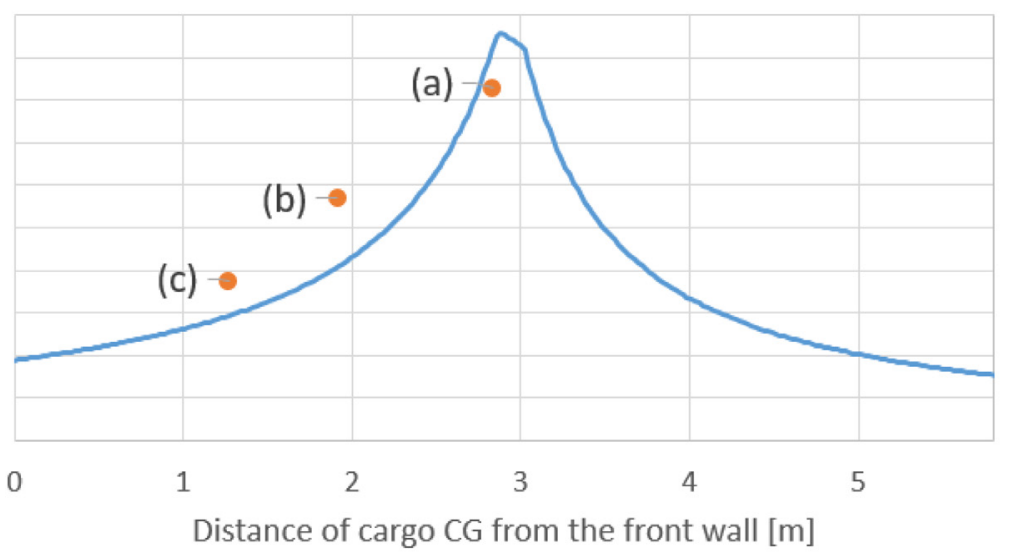

20

18

16

14

12 Cargo

10 Weight

8 [tnf]

(d) LDD

Fig. 1. Solution instance BR.

It is assumed that each box is a rigid body with a centre of gravity located at its geometric centre and that the boxes not only do not overlap but are also placed entirely inside the container and orthogonally to its walls.

The vehicle $V$ is characterised by its load distribution diagrams (LDDs). A LDD is a two-dimensional plot that represents the maximum admissible payload of a vehicle as a function of the longitudinal or transverse position of the centre of gravity (CG) of the cargo, thus defining the area within which the location of the cargo's CG is acceptable, i.e. the load is balanced. A more detailed explanation of the LDDs can be found in Ramos et al. (2018).

Container $C$ is characterised by its depth $(D)$, width $(W)$ and height $(H)$.

A cargo arrangement $P$ assigned to a distribution route is considered load balanced if its load is balanced throughout the complete trip, i.e. after visiting a customer $k$ and delivering the requested set of boxes $K$, the remaining cargo arrangement $P \backslash K$ to be delivered to the following customers is load balanced in the vehicle.

If a given cargo arrangement is not balanced, it is possible to balance it by removing or moving boxes. Box movement has to respect the following constraints:

- a box can only be moved if all boxes above and in front of it are also moved, i.e. there is no horizontal and vertical obstruction to access the box;

- box rotation is allowed.

An example of a three-axle truck longitudinal LDD, loaded with cargo for three customers, is provided in Fig. 1. When the truck is fully loaded with the cargo for the three customers, the plotted location of the cargo's weight versus centre of gravity $\left(C G_{x}\right)$ is inside the admissible region defined by the LDD. However, as the cargo is delivered to each customer, the point moves outside the feasible region, i.e. it is no longer feasible and a cargo rearrangement in the vehicle is required.

\section{Literature review}

Pollaris et al. (2015) presents state-of-the-art and future research directions for the VRP with loading constraints. The authors stress that the incorporation of loading constraints into the VRP is critical in order to achieve a more efficient route planning and that disregarding these constraints impairs planning and induces additional costs with last minute changes.

The incorporation of loading constraints into the VRP has been receiving increased attention from researchers in the last years, with almost $60 \%$ of the papers on this subject being published after 2009. In the literature review proposed by Pollaris et al. (2015), the classification of Bortfeldt and Wäscher (2013) is considered to identify and classify the loading 
constraints. The loading constraints are divided into container-related constraints, which refer to the container or the vehicle where the items are placed, item-related constraints, which refer to individual items, and load-related constraints, which represent constraints concerning the result of the packing process.

Weight limit and weight distribution constraints belong to the category of container-related constraints. Regarding the combination of the VRP with the weight distribution constraint, Pollaris et al. (2015) only identifies the work of Øvstebø et al. (2011) in a maritime transport problem, in which the stability of the ship is guaranteed by considering the torque from the cargo on the ship and by imposing limits to the distance from the ship's bottom deck to its centre of gravity. The integration of axle weight limits into road transport is only considered in Pollaris et al. (2016), which proposes a mixed integer linear programming model in order to solve a vehicle routing problem with sequence-based pallet loading (a 2D packing problem) by incorporating axle weight constraints. The items are homogeneous pallets and can be packed in two horizontal rows in the vehicle. The model considers the axle weight constraint at the depot as well as after each delivery. The computational experiments demonstrate that the model performs adequately for distribution networks of up to 20 customers and that the integration of axle weight constraints into vehicle routing models is required for a feasible route planning. More recently, the same problem is tackled in Pollaris et al. (2017), but an iterated local search algorithm is used to cope with networks of 50-100 customers.

The impact of weight variation along the route is also an important issue in routing problems, e.g. in Tricoire and Parragh (2017), a model used to minimise vehicle emissions and energy consumption is employed in order to address the green city hub location routing problem and in Zachariadis et al. (2015), the weight of the transported cargo has a significant impact in the objective function in the load-dependent vehicle routing problem.

According to Bortfeldt and Wäscher (2013), the maximum weight limit in the CLP has been considered in 14\% of the published papers, such as Gehring and Bortfeldt (1997), Liu and Hsiao (1997), Iori and Martello (2010), Chan et al. (2006), Egeblad et al. (2010) and Wang et al. (2010). The maximum weight that the axles of the vehicles can sustain has also been considered in the CLP literature in Lim et al. (2013) and Alonso et al. (2016, 2017).

The load balance constraint has also been considered in algorithms for the CLP but has been mostly treated as a soft constraint. This important constraint is inherently related to the position of a loaded cargo's centre of gravity. Different strategies have been adopted in the literature, all having in common the geometric midpoint of the base of the container as the desirable location for the centre of gravity of the cargo. The longitudinal load balance in the CLP is considered in Chen et al. (1995) and Lim et al. (2013) and the transverse load balance is considered in Haessler and Brian Talbot (1990); Gehring et al. (1990); Davies and Bischoff (1999); Bortfeldt and Gehring (2001); Techanitisawad and Tangwiwatwong (2004); Moon and Nguyen (2014). There is also some research that takes into account all three dimensions: longitudinal, transverse and vertical load balance, namely Fasano (2004), Egeblad and Pisinger (2009), Liu et al. (2011), Baldi et al. (2012), Costa and Captivo (2016), Alonso et al. (2017) and Paquay et al. (2018).

Ramos et al. (2018) proposes a different approach, complying with real-world transport regulations and legislation. This work considers load balance as a hard constraint and uses vehicle-specific diagrams (the load distribution diagrams), which define the feasibility domain for the location of the centre of gravity of the cargo, according to the specific technical characteristics of the vehicle. It develops a multi-population biased random-key genetic algorithm for the CLP, considering static stability and load balance in the fitness function, thus ensuring load balanced solutions.

In this paper, we first propose a MILP model that recovers the load balance of a cargo arrangement by rearranging and/or removing boxes, having as priority keeping the same service level to customers (a box removed from the cargo is a box not delivered to the customer). Secondly, we present an algorithm that, when provided with a container loading solution, foresees the load balance of an entire route and, if later on, an unbalanced cargo can only be avoided by removing boxes, removes these from the initial load of the truck.

Incorporating load balance constraints into VRP algorithms is crucial for transport industries, which can incur high fines for disregarding legal limitations. This has been receiving increased attention from the European Commission (European Best Practice Guidelines on Cargo Securing for Road Transport). Concerning research, this is a very challenging task and this work will contribute to this goal by ensuring that a cargo arrangement is load balanced along the complete distribution route.

\section{Mixed integer linear programming model for load balance recovery}

The problem of ensuring the load balance of a given cargo arrangement $P$ transported in a given vehicle $V$ is formulated as a mixed integer linear programming (MILP) model. The load balance is enforced by imposing that the cargo's centre of gravity lies within the vehicle's LDD. The total number of boxes is defined as $n$, and the boxes are indexed by $i, i=1, . ., n$. Each box has a depth, width, height and weight $\left(d_{i}, w_{i}, h_{i}, w g t_{i}\right)$, measured along the axis of the corresponding container, and has an initial position $\left(x_{i}^{i n i}, y_{i}^{i n i}, z_{i}^{i n i}\right)$ in the cargo arrangement $P$. Set $T_{i}$ is composed by the indices $j$ of all boxes above box $i$ in $P$, set $F_{i}$ contains the indices $j$ of all boxes in front of box $i$ in $P$ and set $R_{i}$ is composed by the indices $j$ of all boxes to the right of box $i$ in $P$.

The main binary decision variables $\alpha_{i}$ and $\phi_{i}$ are defined for each box $i, i=1, \ldots, n$ and stand for whether box $i$ changes its initial position in the cargo arrangement or is removed from the cargo arrangement, respectively. The coordinates of the insertion position of box $i$ are given by the linear decision variable $x_{i}$, along the depth, $y_{i}$, along the width, and $z_{i}$, along the height. Binary variables $\Theta_{i j}^{x}, \Theta_{i j}^{y}, \Theta_{i j}^{z}$ are auxiliary variables that indicate the relative position of boxes $i$ and $j$. If $j$ is ahead of $i, \Theta_{i j}^{x}$ takes the value 1 , if $j$ is to the left of $i$ it is $\Theta_{i j}^{y}$ that takes the value 1 , and, finally, if $j$ is above $i$, $\Theta_{i j}^{z}$ takes the 
value 1. Regarding the position of the centre of gravity of the loaded cargo, variable $C_{x}$ contains the coordinate along the container's depth direction and variable $C_{y}$ the coordinate along the container's width direction. Variable $P_{\text {cargo }}$ stands for the total weight of the cargo loaded.

For the sake of clarity, the formulation (1)-(40) is divided and explained in three subsections:

1. Objective function and movement constraints - The main objective is to minimise the number of boxes moved and/or removed, the latter being the recourse option to balance cargo $P$. The movement constraints ensure that if a box changes its initial position, each box that is above and in front of it also moves, changing its position and guaranteeing access to the first box. If a box is not moved or removed from $P$, its position remains unchanged.

2. Geometric constraints - This set of constraints ensures that the boxes do not overlap and that boxes are completely inside the vehicle's container.

3. Load balance constraints - Lastly, load balance constraints are introduced. These constraints are strongly related to the LDD, whose boundary gives the maximum permissible payload of a vehicle as a function of the position of the cargo's centre of gravity.

\subsection{Objective function and movement constraints}

Each stop of the vehicle to deliver cargo to a customer should be as fast as possible and so the number of boxes moved to load balance $P$ should be minimal. Objective function (1) intends to minimise the number of boxes removed from the container and the number of boxes moved inside the container. It is better to move boxes than to remove them from the loaded cargo, which is only done when this is mandatory in order to achieve a balanced cargo.

Minimise $\mathrm{Z}=\sum_{i=1}^{n}(n+1) \cdot \phi_{i}+\alpha_{i}$

Subject to: $1-\alpha_{i}-\alpha_{j} \leq \Theta_{i j}^{x}, \quad \forall i=1, \ldots, n ; j \in F_{i}$,

$$
\begin{aligned}
& 1-\alpha_{i}-\alpha_{j} \leq \Theta_{i j}^{y}, \quad \forall i=1, \ldots, n ; j \in R_{i}, \\
& 1-\alpha_{i}-\alpha_{j} \leq \Theta_{i j}^{z}, \quad \forall i=1, \ldots, n ; j \in T_{i}, \\
& \sum_{j \in F_{i}}^{n}\left(1-\alpha_{j}\right) \leq\left|F_{i}\right| \cdot\left(1-\alpha_{i}\right), \quad \forall i=1, \ldots, n, \\
& \sum_{j \in T_{i}}^{n}\left(1-\alpha_{j}\right) \leq\left|T_{i}\right| \cdot\left(1-\alpha_{i}\right), \quad \forall i=1, \ldots, n, \\
& x_{i}+D \cdot\left(1-\alpha_{i}\right) \leq x_{i}^{i n i}+D, \quad \forall i=1, \ldots, n, \\
& x_{i}-D \cdot\left(1-\alpha_{i}\right) \geq x_{i}^{i n i}-D, \quad \forall i=1, \ldots, n, \\
& y_{i}+W \cdot\left(1-\alpha_{i}\right) \leq y_{i}^{i n i}+W, \quad \forall i=1, \ldots, n, \\
& y_{i}-W \cdot\left(1-\alpha_{i}\right) \geq y_{i}^{i n i}-W, \quad \forall i=1, \ldots, n, \\
& z_{i}+H \cdot\left(1-\alpha_{i}\right) \leq z_{i}^{i n i}+H, \quad \forall i=1, \ldots, n, \\
& z_{i}-H \cdot\left(1-\alpha_{i}\right) \geq z_{i}^{i n i}-H, \quad \forall i=1, \ldots, n, \\
& x_{i}+D \cdot \phi_{i} \leq x_{i}^{i n i}+D, \quad \forall i=1, \ldots, n,
\end{aligned}
$$




$$
\begin{aligned}
& x_{i}-D \cdot \phi_{i} \geq x_{i}^{i n i}-D, \quad \forall i=1, . ., n, \\
& y_{i}+W \cdot \phi_{i} \leq y_{i}^{i n i}+W, \quad \forall i=1, . ., n, \\
& y_{i}-W \cdot \phi_{i} \geq y_{i}^{i n i}-W, \quad \forall i=1, . ., n, \\
& z_{i} \geq H \cdot \phi_{i}, \quad \forall i=1, . ., n, \\
& \phi_{i} \leq \alpha_{i}, \quad \forall i=1, . ., n,
\end{aligned}
$$

Constraints (2) ensure that if box $j$ is in front of box $i$ in the initial cargo arrangement, and if the $i$ and $j$ boxes are not moved, box $j$ stays ahead of box $i$. Similarly, constraint (3) ensures that if box $j$ is to the right of box $i$ in the initial cargo arrangement, and if the $i$ and $j$ boxes are not moved, box $j$ stays to the right of box $i$. Similarly, constraint (4) ensures that if box $j$ is above box $i$ in the initial cargo arrangement and if the $i$ and $j$ boxes are not moved, box $j$ stays above box $i$.

Constraint (5) ensures that if box $i$ is moved, every box that is in front of box $i$ in the initial cargo arrangement is also moved. Similarly, in constraint (6), if box $i$ is moved then every box that is above box $i$ in the initial cargo arrangement is also moved.

The set of constraints (7)-(12) ensures that if box $i$ is not moved, it maintains the position of the initial cargo arrangement.

The set of constraints (13)-(17) ensures that if box $i$ is removed from the initial cargo arrangement, it will maintain the position in $x$ and $y$ and will be positioned virtually outside the container, on its top $z_{i} \geq H$.

Constraints (18) ensure that if box $i$ is removed from the cargo arrangement, it will also be marked as moved.

\subsection{Geometric constraints}

The following set of constraints ensures that the boxes do not overlap in the balanced arrangement $P$ and lie entirely within the container.

$$
\begin{aligned}
& x_{i}+d_{i} \leq x_{j}+\left(1-\Theta_{i j}^{x}\right) \cdot D, \quad \forall i=1, \ldots, n ; j=1, . ., n ; i \neq j, \\
& y_{i}+w_{i} \leq y_{j}+\left(1-\Theta_{i j}^{y}\right) \cdot W, \quad \forall i=1, \ldots, n ; j=1, \ldots, n ; i \neq j, \\
& z_{i}+w_{i} \leq z_{j}+\left(1-\Theta_{i j}^{z}\right) \cdot H \quad \forall i=1, \ldots, n ; j=1, \ldots, n ; i \neq j, \\
& \Theta_{i j}^{x}+\Theta_{j i}^{x}+\Theta_{i j}^{y}+\Theta_{j i}^{y}+\Theta_{i j}^{z}+\Theta_{j i}^{z} \geq 1 \quad \forall i=1, \ldots, n ; j=1, \ldots, n ; i \neq j, \\
& \Theta_{i j}^{x}+\Theta_{j i}^{x} \leq 1, \quad \forall i=1, \ldots, n ; j=i, . ., n ; i \neq j, \\
& \Theta_{i j}^{y}+\Theta_{j i}^{y} \leq 1, \quad \forall i=1, \ldots, n ; j=i, . ., n ; i \neq j, \\
& \Theta_{i j}^{z}+\Theta_{j i}^{z} \leq 1, \quad \forall i=1, \ldots, n ; j=i, . ., n ; i \neq j, \\
& x_{i} \leq D-d_{i}, \quad \forall i=1, . ., n, \\
& y_{i} \leq W-w_{i}, \quad \forall i=1, . . n, \\
& z_{i} \leq H-h_{i}+H \cdot \phi_{i}, \quad \forall i=1, . ., n,
\end{aligned}
$$

The geometric constraints of box no-overlap is ensured by constraints (19)-(25). Through the relative position between each pair of boxes $i$ and $j$, it is possible to ensure that the boxes do not overlap, regardless of the box maintaining or changing its initial position. Constraints (26)-(28) ensure that if box $i$ is not removed from the cargo arrangement, it should be inside the container. 

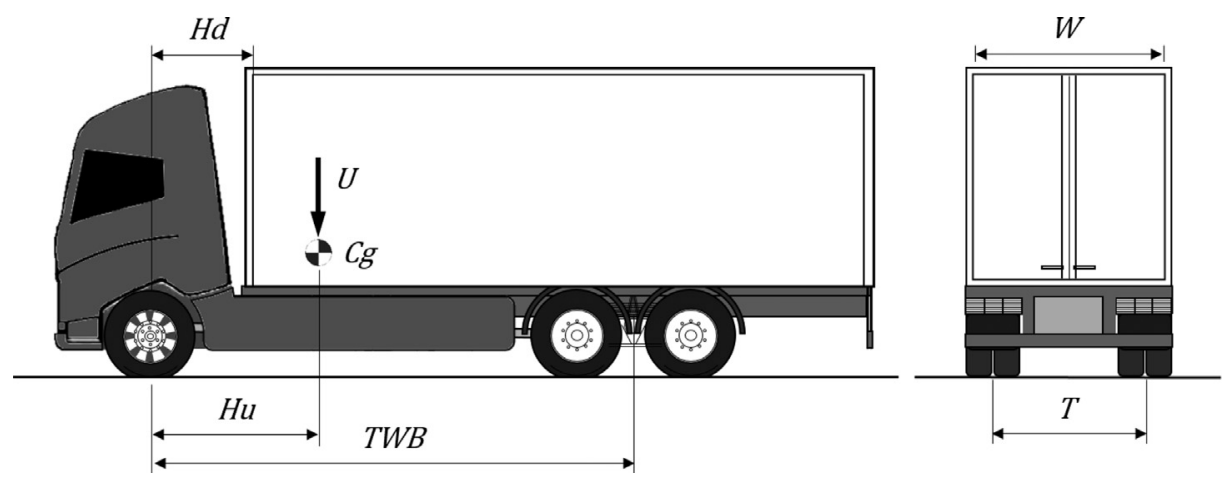

Fig. 2. Vehicle data.

\subsection{Load balance constraints}

The following set of constraints ensure that the cargo's CG, along the depth $\left(C_{x}\right)$ and width $\left(C_{y}\right)$ of the container, is located within the feasibility domain, defined by the respective LDDs that take into account the real characteristics of the vehicle.

A LDD is a boundary curve that expresses the maximum permissible payload of a vehicle as a function of the position of its centre of gravity along a given dimension (longitudinal or transverse), measured from the origin of the container. A more detailed explanation of LDDs is presented in Ramos et al. (2018).

The data required for the calculation of a LDD is of technical and regulatory nature. The first includes the vehicle data and concerns its mechanical characteristics (Fig. 2); the latter concerns the legislation that regulates the maximum authorised dimensions and weights for road vehicles. These values are specific to each country, and in the EU this issue is regulated by the Council Directive 96/53/EC of 25 July 1996 and the Directive (EU) 2015/719.

The data required for the calculation of the LDD of a vehicle is:

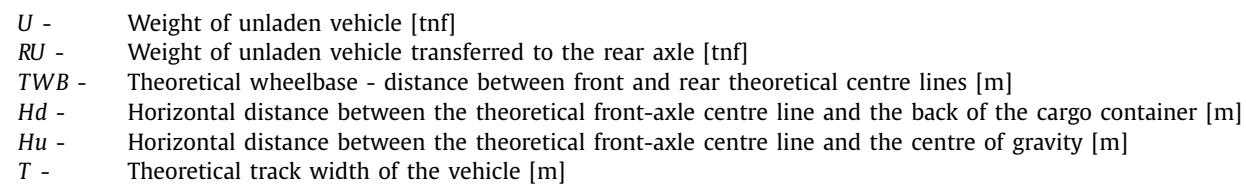

The regulatory requirements for the calculation of a LDD are:

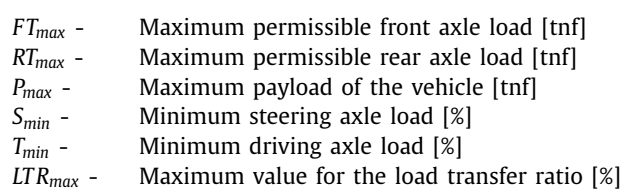

All these parameters are used in the following constraints:

$$
\begin{aligned}
& C_{x}=\sum_{i=1}^{n} \frac{\left(2 x_{i}+d_{i}\right) \cdot w g t_{i}}{2}-\frac{\left(2 x_{i}^{i n i}+d_{i}\right) \cdot w g t_{i}}{2} \cdot \phi_{i}, \\
& C_{y}=\sum_{i=1}^{n} \frac{\left(2 y_{i}+w_{i}\right) \cdot w g t_{i}}{2}-\frac{\left(2 y_{i}^{i n i}+w_{i}\right) \cdot w g t_{i}}{2} \cdot \phi_{i}, \\
& P_{\text {cargo }}=\sum_{i=1}^{n} w g t_{i} \cdot\left(1-\phi_{i}\right), \\
& C_{x} \geq P_{\text {cargo }} \cdot(T W B-H d)-T W B \cdot F T_{\text {max }}-U \cdot(T W B-H u), \\
& C_{x} \leq T W B \cdot R T_{\text {max }}-U \cdot H u-P_{\text {cargo }} \cdot H d, \\
& C_{x} \leq U \cdot\left(T W B-H u-S_{\min } \cdot T W B\right)-P_{\text {cargo }} \cdot\left(S_{\min } \cdot T W B-H u+T W B\right),
\end{aligned}
$$




$$
\begin{aligned}
& C_{x} \geq U \cdot\left(T_{\min } \cdot T W B-H u\right)-P_{\text {cargo }} \cdot\left(H d-T_{\min } \cdot T W B\right), \\
& C_{y} \geq\left(-U \cdot T \cdot L T R_{\max }-P_{\text {cargo }} \cdot\left(T \cdot L T R_{\max }-W\right)\right) \cdot \frac{1}{2}, \\
& C_{y} \leq\left(-U \cdot T \cdot L T R_{\max }+P_{\text {cargo }} \cdot\left(-T \cdot L T R_{\max }-W\right)\right) \cdot\left(\frac{-1}{2}\right), \\
& P_{\text {cargo }} \leq P_{\max }, \\
& \alpha_{i}, \phi_{i}, \Theta_{i j}^{x}, \Theta_{i j}^{y}, \Theta_{i j}^{z} \in\{0,1\}, \quad i=1, \ldots, n ; j=1, \ldots, n, \\
& C_{x}, C_{y}, P_{\text {cargo }}, x_{i}, y_{i}, z_{i} \geq 0 \quad i=1, . ., n .
\end{aligned}
$$

Constraints (29) and (30) are the numerator of the quotient of the formulae for the centre of gravity in $x$ and $y$ respectively. The total weight of the cargo is calculated in constraint (31).

Constraint (32) represents the maximum permissible front axle load using the equation of moments about the rear axle and the total weight of the cargo loaded.

Similarly, constraint (33) represents the maximum permissible rear axle load using the equation of moments about the front axle and the total weight of the cargo loaded.

The minimum permissible steering axle load is guaranteed by constraint (34) using the equation of moments about the rear axle and the total weight of the cargo loaded and constraint (35) ensures the minimum driving/traction axle load using the equation of moments about the front axle and the total weight of the cargo loaded.

In a transverse LDD, the transverse position of the cargo's CG is evaluated in relation to the vertical symmetry plane of the vehicle. The maximum permissible load difference between the left and right wheels is ensured by constraints (36) and (37), which use the equation of moments about the midpoint of the tyre footprint and the total weight of the cargo loaded.

The maximum cargo weight is limited by constraint (38). Constraints (39) and (40) define the feasibility domains for the decision variables.

\section{Multi-drop load balance recovery algorithm}

This section proposes a complete solution to the multi-drop load balance recovery problem. The multi-drop load balance recovery algorithm (MDLBRA) ensures that given a cargo arrangement $P$, loaded in a container $C$, and transported by vehicle $V$, it is possible to modify the arrangement so that it remains balanced when the cargo is partially unloaded at the various customers' destinations. The MDLBRA removes the boxes that would render the cargo balancing unfeasible during the trip from the cargo arrangement at the depot. It also indicates which boxes must be moved and their new positions in $C$ after unloading the cargo at a customer and before continuing the trip, ensuring cargo load balance in the complete route.

The MDLBRA is presented in Fig. 3.

The algorithm iteratively analyses the cargo arrangement along its route. It starts at the depot $(k=0)$, with the full cargo for all customers. It runs the proposed MILP model to balance the cargo. If the MILP model is not able to find a feasible solution within the time limit, the Recovery Heuristic (RH), which removes boxes from the arrangement until a balanced cargo is achieved, is applied (the heuristic is fully described in the next subsection).

The MDLBRA continues analysing the trip, verifying through the MILP model if the cargo arrangement remains balanced after removing the boxes that have to be unloaded at the next customer. If a balanced situation is achieved by only changing box positions, the algorithm continues by analysing the following customer, until the last customer is reached. However, each time the MILP model reaches a solution that requires the removal of a box, or the RH has to be used, the MDLBRA backtracks the analysis to the depot, removes the boxes that would not allow cargo balance during the complete trip from the configuration, and restarts the analysis from the depot with the new configuration.

\subsection{Recovery Heuristic}

The Recovery Heuristic ( $\mathrm{RH})$ is used when the MILP model is not able to find a feasible solution within a defined time limit. The RH is a heuristic that enforces cargo balance, given a cargo arrangement and a transport vehicle. Using the CG of a cargo arrangement and the LDD of the vehicle, the algorithm iteratively selects one box to be removed from the arrangement, so that a balance is achieved. The box with the highest product of "distance of the box's centre of gravity to the origin of the coordinates" $\times$ "box density" (measured as the ratio weight over volume) (41) is selected. The goal is to remove the 


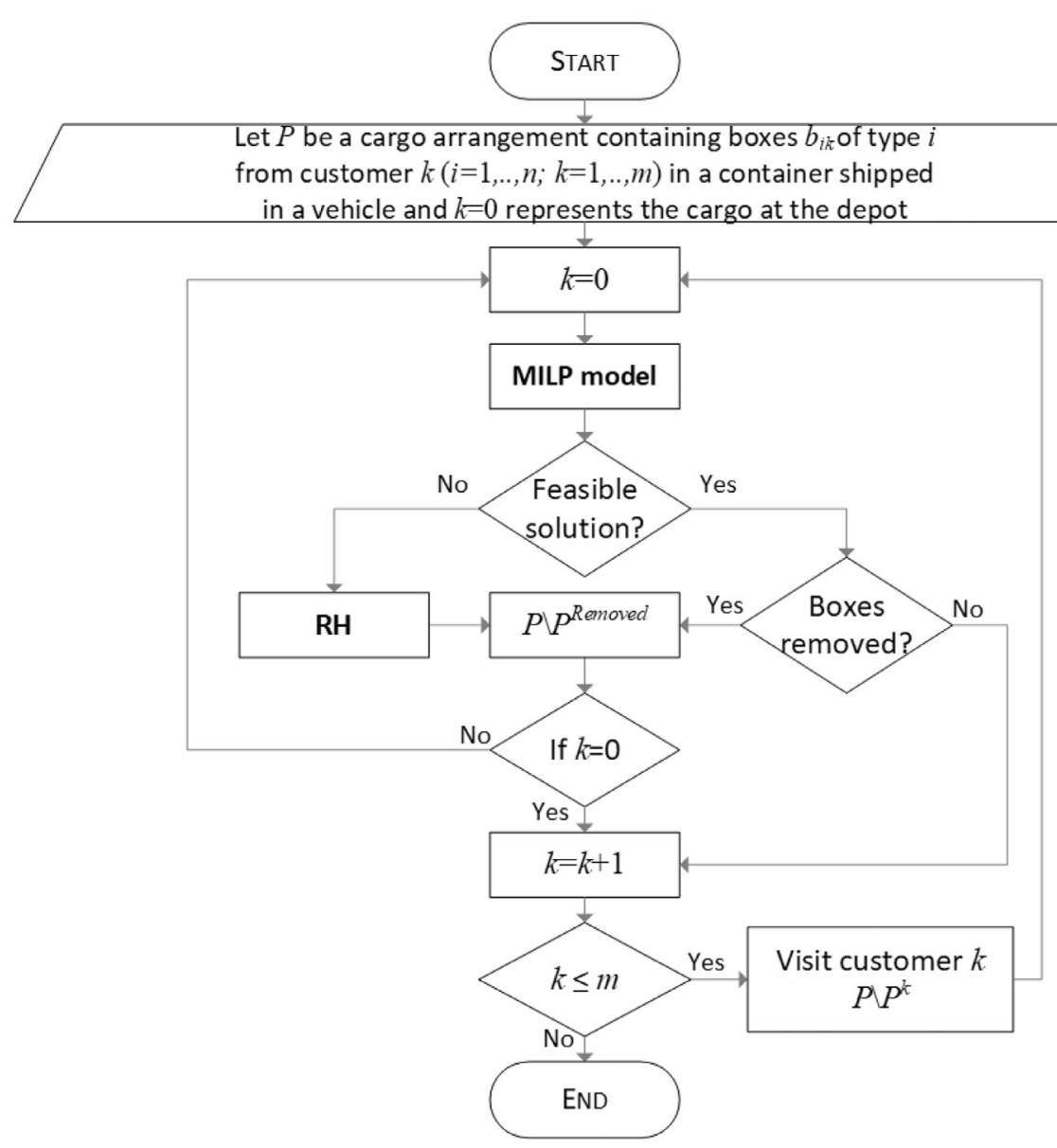

Fig. 3. MDLBRA flowchart.

smallest volume of boxes that have the biggest impact in the (un)balancing: the heaviest ones closer to the vehicle's rear. The pseudo code of the Recovery Heuristic is described in Algorithm 1.

$$
f_{i}=\frac{C G_{i} \times w g t_{i}}{v_{i}}
$$

\section{Computational results for the MDLBRA}

The MDLBRA was implemented in C++ in Microsoft Visual Studio with solver IBM ILOG Cplex 12.63. The computational experiments were run on an Intel Xeon CPU E5-2687W $03.10 \mathrm{GHz}$ with 128GB of RAM, with a time limit of $600 \mathrm{~s}$.

Extensive computational experiments have been conducted on a set of test problems taken from the literature. In Ramos et al. (2018), the test instances most known and used in computational experiments with container loading algorithms, the BR data sets proposed by Bischoff and Ratcliff (1995) and Davies and Bischoff (1999), were extended to consider the weight of the boxes. BR1 to BR15 problem instances are organised into 15 classes, with a total of 100 instances per class. The heterogeneity of the boxes increases from just 3 different box types (in BR1) to 100 box types (in BR15).

Additionally, to tackle load balance, the characteristics of the vehicle are also required. Ramos et al. (2018) used the characteristics of the "'Volvo Truck FE 62TR Air Ride" truck (Volvo Trucks, 2015), since the dimensions of this vehicle are compatible with the dimensions of the container used in the BR test problems $(L=587 \mathrm{~cm}, W=233 \mathrm{~cm}$ and $H=220 \mathrm{~cm})$. Table 1 presents the vehicle data.

In what concerns the cargo arrangements, the solutions of the BRKGA algorithm proposed by Ramos et al. (2016) for the container loading problem with static stability constraints were used. However, the cargo arrangement has to be evaluated each time a customer is visited during the distribution trip. To emulate the demand of the different customers to be visited, each cargo arrangement was divided into three subsets. The first includes the boxes with $x_{1}$ coordinate less than $50 \%$ of the length of the container (customer 3), the second with less than 75\% (customers 2 and 3 ) and the third with the complete solution (customers 1, 2 and 3). Therefore, a total of 4500 cargo arrangements (15 classes, 100 instances per class and 3 customers per instance) were evaluated. 


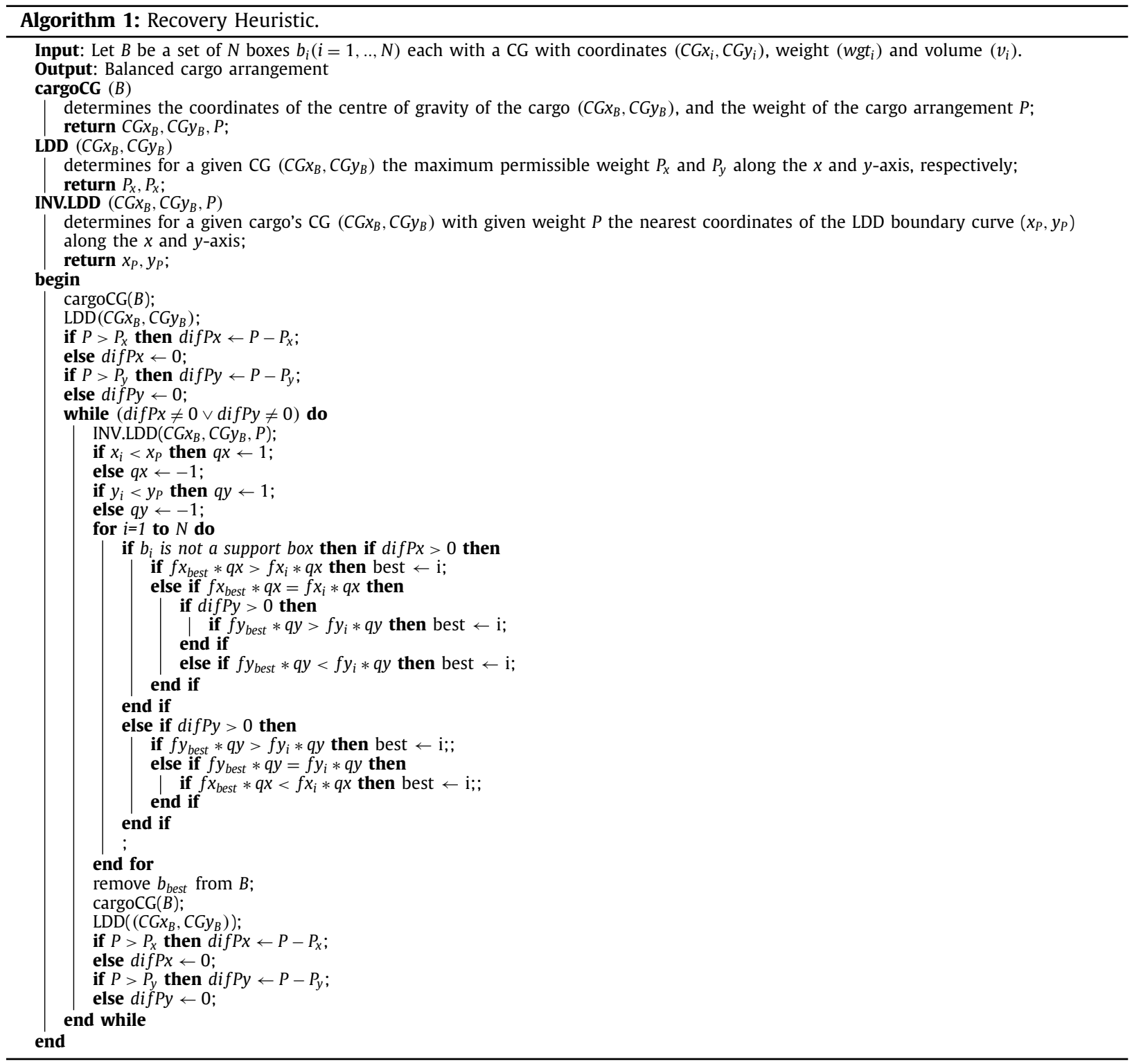

Table 1

Vehicle parameters.

\begin{tabular}{|c|c|c|c|}
\hline Parameters & Values & Parameters & Values [ton-force] \\
\hline Hd & $0.832 \mathrm{~m}$ & $U$ & 6.840 \\
\hline$T W B$ & $4.397 \mathrm{~m}$ & $R U$ & 2.800 \\
\hline$T$ & $1.831 \mathrm{~m}$ & $P_{\max }$ & 19.160 \\
\hline$T_{\min }$ & $25 \%$ & $R T_{\max }$ & 19.000 \\
\hline$S_{\min }$ & $25 \%$ & $F T_{\max }$ & 7.100 \\
\hline$L T R_{\max }$ & $10 \%$ & & \\
\hline
\end{tabular}

Fig. 4 presents the solution for instance BR_15_4 when just the cargo of the last customer to be visited in the route (customer 3 ) is under consideration. Fig. 4a represents the unbalanced cargo arrangement, and the boxes that will be moved are highlighted. Fig. 4b shows the load balanced solution obtained by the MILP model.

This is not a container loading algorithm. Instead, the goal is to modify solutions generated by any container loading algorithm so that load balance is guaranteed when the truck leaves the depot and during the entire route. Solutions for the CLP and the corresponding routes are therefore needed as the initial data for the approach described in this paper. 


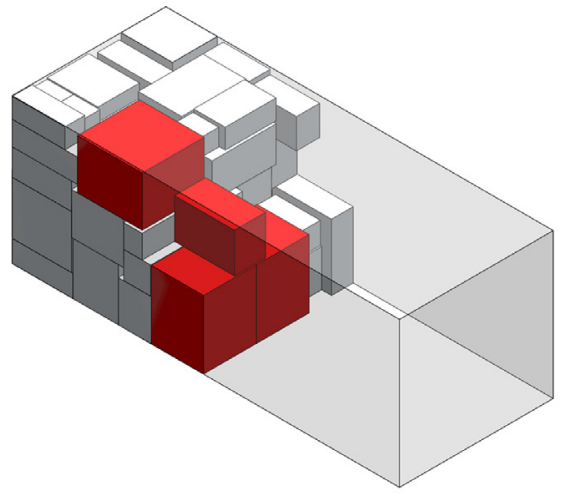

(a) Unbalanced Solution

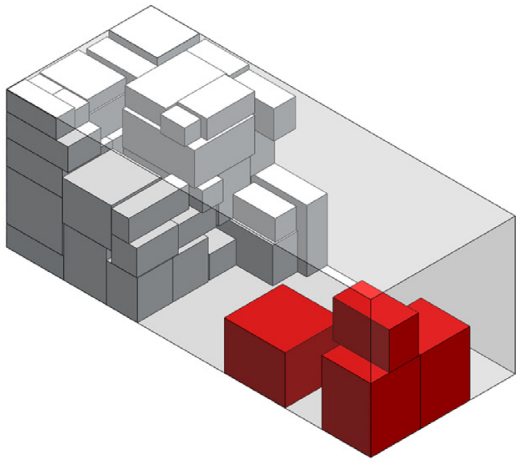

(b) Balanced Solution

Fig. 4. Solution instance BR.

Table 2

Computational results for the cargo arrangement with the cargo of customers 1, 2 and 3.

\begin{tabular}{|c|c|c|c|c|c|c|c|c|c|c|c|c|c|}
\hline \multicolumn{5}{|l|}{ Instances } & \multicolumn{7}{|c|}{ MILP model } & \multicolumn{2}{|c|}{ Recovery Heuristic } \\
\hline Class & \#Items & Weight & Vol & \#Unb & \#Opt & \#Feas & \#Unk & \#Moved & \#Removed & GAP & Time & \#Sols & \#Removed \\
\hline BR 1 & 142.4 & 17981.6 & 94.8 & 43 & 5 & 10 & 28 & 17.8 & 2.7 & 37.2 & 542.1 & 28 & 18.7 \\
\hline BR 2 & 129.1 & 18179.0 & 95.4 & 45 & 6 & 11 & 28 & 19.1 & 3.4 & 72.8 & 545.2 & 28 & 15.1 \\
\hline BR 3 & 126.9 & 18465.9 & 95.5 & 54 & 9 & 23 & 22 & 28.0 & 2.4 & 60.3 & 530.7 & 22 & 16.0 \\
\hline BR 4 & 124.5 & 18342.6 & 95.5 & 53 & 3 & 26 & 24 & 26.3 & 2.8 & 59.5 & 581.1 & 24 & 12.9 \\
\hline BR 5 & 124.9 & 17956.9 & 95.4 & 44 & 0 & 23 & 21 & 32.1 & 1.7 & 69.3 & 602.0 & 21 & 11.6 \\
\hline BR 6 & 122.4 & 18183.2 & 95.4 & 51 & 2 & 24 & 25 & 26.8 & 2.3 & 61.4 & 584.5 & 25 & 10.8 \\
\hline BR 7 & 120.9 & 17926.9 & 95.1 & 39 & 2 & 18 & 19 & 19.6 & 1.6 & 74.2 & 577.9 & 19 & 8.7 \\
\hline BR 8 & 120.2 & 17973.3 & 94.7 & 43 & 4 & 26 & 13 & 23.2 & 1.1 & 60.1 & 554.9 & 13 & 8.1 \\
\hline BR 9 & 117.9 & 17860.6 & 94.5 & 28 & 0 & 19 & 9 & 36.0 & 1.7 & 56.3 & 579.3 & 9 & 7.6 \\
\hline BR 10 & 118.1 & 17928.5 & 94.0 & 26 & 1 & 15 & 10 & 26.4 & 0.6 & 84.0 & 581.6 & 10 & 6.2 \\
\hline BR 11 & 116.4 & 17976.9 & 93.5 & 28 & 0 & 13 & 15 & 19.2 & 0.8 & 57.2 & 603.6 & 15 & 5.1 \\
\hline BR 12 & 116.1 & 17797.4 & 92.8 & 26 & 3 & 12 & 11 & 23.8 & 0.5 & 63.0 & 538.8 & 11 & 3.9 \\
\hline BR 13 & 114.7 & 17431.1 & 91.7 & 19 & 1 & 7 & 11 & 42.4 & 0.5 & 87.2 & 582.4 & 11 & 4.6 \\
\hline BR 14 & 113.3 & 17434.4 & 91.0 & 18 & 0 & 14 & 4 & 29.0 & 0.3 & 76.9 & 600.5 & 4 & 3.3 \\
\hline BR 15 & 111.8 & 17168.2 & 89.7 & 4 & 0 & 3 & 1 & 61.7 & 0.0 & 95.0 & 601.1 & 1 & 2.0 \\
\hline BR (1-7) & 127.3 & 18148.0 & 95.3 & 47 & 4 & 19 & 24 & 24.2 & 2.4 & 62.1 & 566.2 & 24 & 13.4 \\
\hline BR (8-15) & 116.1 & 17696.3 & 92.7 & 24 & 1 & 14 & 9 & 32.7 & 0.7 & 72.5 & 580.3 & 9 & 5.1 \\
\hline BR (1-15) & 121.3 & 17907.1 & 93.9 & 35 & 2 & 16 & 16 & 28.8 & 1.5 & 67.6 & 573.7 & 16 & 9.0 \\
\hline
\end{tabular}

Tables 2-4 present the computational results for the three subsets. The first five columns describe the instances, column (\#Items) displays the average number of boxes in the cargo arrangement for each CLP solution of the BR class, column (Weight) presents the average initial weight in $\mathrm{kg}$, and column (Vol) shows the initial average percentage of container volume used. Column (\#Unb) presents the total number of unbalanced instances for each class.

The following six columns describe the solutions obtained with the MILP model: column (\#Opt) presents the number of optimal solutions found by Cplex; column (\#Feas) displays the total number of solutions in which the solver reached the time limit with a feasible solution; and column(\#Unk) presents the number of instances where the solver reached the time limit without a feasible solution. Column (\#Moved) presents the average number of boxes that were moved in the cargo arrangement for each class and column (\#Removed) shows the average number of boxes removed from the cargo arrangement. Column (GAP) presents the average gap for the feasible solutions and the last column displays the average computational time in seconds (Time).

The last two columns focus on the solutions obtained with the RH for the problem instances in which the solver was not able to find a feasible solution with the MILP model. Column (\#Sols) displays the number of instances solved by the RH and column (\#Removed) presents the average number of boxes removed from the cargo arrangement by the RH.

For the cargo arrangements, considering the orders of all customers (100\% of the cargo), 521 out of 1500 instances were unbalanced. It is worth noting that the number of unbalanced solutions by class instance decreases as heterogeneity increases. A possible explanation is that as heterogeneity increases, the volume occupation rate decreases, which means that total cargo weight also decreases, leading to an increase of the LDD area where the centre of gravity of the cargo can be located. For this same subset of test instances, the solver was only able to provide an optimal solution for $7 \%$ of the unbalanced test instances, whereas for $47 \%$ of the instances the time limit was reached with a feasible solution and for the 
Table 3

Computational results for the cargo arrangement with the cargo of customers 2 and 3 .

\begin{tabular}{|c|c|c|c|c|c|c|c|c|c|c|c|c|c|}
\hline \multicolumn{5}{|l|}{ Instances } & \multicolumn{7}{|c|}{ MILP model } & \multicolumn{2}{|c|}{ Recovery Heuristic } \\
\hline Class & \#Items & Weight & Vol & \#Unb & \#Opt & \#Feas & \#Unk & \#Moved & \#Removed & GAP & Time & \#Sols & \#Removed \\
\hline BR 1 & 110.3 & 14166.9 & 75.4 & 90 & 65 & 25 & 0 & 26.2 & 0.1 & 52.7 & 210.4 & 0 & 0.0 \\
\hline BR 2 & 99.0 & 14271.0 & 75.6 & 88 & 72 & 16 & 0 & 9.4 & 0.0 & 37.5 & 159.8 & 0 & 0.0 \\
\hline BR 3 & 95.7 & 14660.4 & 76.3 & 96 & 72 & 23 & 1 & 11.7 & 0.0 & 41.6 & 210.2 & 1 & 17.0 \\
\hline BR 4 & 93.2 & 14659.1 & 76.6 & 97 & 75 & 22 & 0 & 11.4 & 0.0 & 38.4 & 206.5 & 0 & 0.0 \\
\hline BR 5 & 94.0 & 14449.7 & 76.9 & 99 & 73 & 26 & 0 & 11.4 & 0.0 & 37.6 & 222.6 & 0 & 0.0 \\
\hline BR 6 & 91.4 & 14540.3 & 76.7 & 95 & 67 & 28 & 0 & 12.7 & 0.0 & 38.8 & 251.1 & 0 & 0.0 \\
\hline BR 7 & 91.0 & 14492.5 & 77.2 & 96 & 72 & 24 & 0 & 11.4 & 0.0 & 39.2 & 218.9 & 0 & 0.0 \\
\hline BR 8 & 90.8 & 14617.0 & 77.2 & 98 & 58 & 40 & 0 & 14.0 & 0.0 & 37.0 & 281.6 & 0 & 0.0 \\
\hline BR 9 & 89.1 & 14600.6 & 77.4 & 99 & 73 & 26 & 0 & 12.4 & 0.0 & 35.3 & 247.0 & 0 & 0.0 \\
\hline BR 10 & 90.1 & 14736.6 & 77.5 & 100 & 61 & 39 & 0 & 15.0 & 0.0 & 36.5 & 319.7 & 0 & 0.0 \\
\hline BR 11 & 88.4 & 14703.9 & 76.6 & 100 & 53 & 47 & 0 & 16.5 & 0.0 & 35.8 & 346.8 & 0 & 0.0 \\
\hline BR 12 & 88.6 & 14703.7 & 76.5 & 99 & 53 & 46 & 0 & 15.8 & 0.0 & 34.5 & 342.8 & 0 & 0.0 \\
\hline BR 13 & 87.8 & 14334.2 & 75.4 & 99 & 61 & 37 & 1 & 13.8 & 0.0 & 31.3 & 293.9 & 1 & 9.0 \\
\hline BR 14 & 87.4 & 14433.4 & 75.2 & 100 & 53 & 47 & 0 & 15.6 & 0.0 & 34.4 & 356.7 & 0 & 0.0 \\
\hline BR 15 & 87.0 & 14230.7 & 74.4 & 100 & 68 & 32 & 0 & 13.6 & 0.0 & 29.8 & 288.1 & 0 & 0.0 \\
\hline BR (1-7) & 96.4 & 14462.8 & 76.4 & 94 & 71 & 23 & 0 & 13.4 & 0.0 & 40.8 & 211.3 & 0 & 2.4 \\
\hline BR (8-15) & 88.6 & 14545.0 & 76.3 & 99 & 60 & 39 & 0 & 14.6 & 0.0 & 34.3 & 309.6 & 0 & 1.1 \\
\hline BR (1-15) & 92.2 & 14506.7 & 76.3 & 97 & 65 & 32 & 0 & 14.1 & 0.0 & 37.4 & 263.7 & 0 & 1.7 \\
\hline
\end{tabular}

Table 4

Computational results for the cargo arrangement with the cargo of customer 3.

\begin{tabular}{|c|c|c|c|c|c|c|c|c|c|c|c|c|c|}
\hline \multicolumn{5}{|l|}{ Instances } & \multicolumn{7}{|c|}{ MILP model } & \multicolumn{2}{|c|}{ Recovery Heuristic } \\
\hline Class & \#Items & Weight & Vol & \#Unb & \#Opt & \#Feas & \#Unk & \#Moved & \#Removed & GAP & Time & \#Sols & \#Removed \\
\hline BR 1 & 77.3 & 9895.7 & 52.7 & 99 & 92 & 4 & 3 & 6.3 & 0.0 & 11.1 & 68.5 & 3 & 20.7 \\
\hline BR 2 & 68.6 & 9884.4 & 52.6 & 99 & 97 & 2 & 0 & 5.7 & 0.0 & 32.9 & 32.4 & 0 & 0.0 \\
\hline BR 3 & 65.5 & 10119.3 & 52.8 & 99 & 96 & 3 & 0 & 6.5 & 0.0 & 19.6 & 41.5 & 0 & 0.0 \\
\hline BR 4 & 64.0 & 10240.7 & 53.5 & 100 & 98 & 2 & 0 & 6.3 & 0.0 & 11.7 & 26.1 & 0 & 0.0 \\
\hline BR 5 & 63.3 & 9991.6 & 53.1 & 100 & 97 & 3 & 0 & 6.9 & 0.0 & 14.9 & 38.7 & 0 & 0.0 \\
\hline BR 6 & 62.0 & 10133.6 & 53.5 & 100 & 93 & 7 & 0 & 7.6 & 0.0 & 9.5 & 66.0 & 0 & 0.0 \\
\hline BR 7 & 61.2 & 10031.2 & 53.5 & 100 & 98 & 2 & 0 & 6.8 & 0.0 & 8.0 & 26.5 & 0 & 0.0 \\
\hline BR 8 & 61.9 & 10254.7 & 54.1 & 100 & 100 & 0 & 0 & 7.5 & 0.0 & 0.0 & 16.3 & 0 & 0.0 \\
\hline BR 9 & 60.5 & 10089.8 & 53.9 & 100 & 99 & 1 & 0 & 7.9 & 0.0 & 5.0 & 18.3 & 0 & 0.0 \\
\hline BR 10 & 61.7 & 10251.7 & 53.8 & 100 & 98 & 2 & 0 & 8.8 & 0.0 & 5.0 & 36.8 & 0 & 0.0 \\
\hline BR 11 & 60.1 & 10259.4 & 53.5 & 100 & 95 & 5 & 0 & 8.7 & 0.0 & 8.3 & 47.5 & 0 & 0.0 \\
\hline BR 12 & 61.0 & 10243.4 & 53.3 & 100 & 96 & 4 & 0 & 8.8 & 0.0 & 7.5 & 38.3 & 0 & 0.0 \\
\hline BR 13 & 61.2 & 10018.0 & 52.6 & 100 & 98 & 2 & 0 & 8.7 & 0.0 & 8.1 & 23.7 & 0 & 0.0 \\
\hline BR 14 & 60.5 & 10086.7 & 52.5 & 100 & 99 & 1 & 0 & 9.0 & 0.0 & 6.7 & 23.9 & 0 & 0.0 \\
\hline BR 15 & 60.0 & 9868.1 & 51.6 & 100 & 99 & 1 & 0 & 8.9 & 0.0 & 7.7 & 20.7 & 0 & 0.0 \\
\hline BR (1-7) & 66.0 & 10042.4 & 53.1 & 100 & 96 & 3 & 0 & 6.6 & 0.0 & 15.4 & 42.8 & 0 & 0.0 \\
\hline BR (8-15) & 60.9 & 10134.0 & 53.2 & 100 & 98 & 2 & 0 & 8.5 & 0.0 & 6.0 & 28.2 & 0 & 0.0 \\
\hline BR (1-15) & 63.2 & 10091.2 & 53.1 & 100 & 97 & 3 & 0 & 7.6 & 0.0 & 10.4 & 35.0 & 0 & 0.0 \\
\hline
\end{tabular}

remaining $46 \%$ the solver did not get a feasible solution. It is interesting to notice that for the instance of class BR 13 for which optimality was proven, the solver only required $34.91 \mathrm{~s}$ to reach the optimum.

Still, when considering the full truck load (the cargo for all customers), in many instances, in order to obtain a feasible load balanced solution, it was necessary to remove boxes from the cargo arrangement, since the maximum weight constraint was not satisfied. The removal of boxes from the cargo arrangements at the depot is not so critical since, at this stage, it can be done before loading the cargo into the vehicle and the boxes removed can be sent in another vehicle. For the 241 test problems without a feasible solution, the RH ensures load balance by removing boxes from the cargo arrangement. The average number of boxes removed from the cargo arrangement by the RH was nine.

Considering the cargo arrangements after visiting customer 1, i.e. with the cargo of customers 2 and 3, 1456 out of 1500 cargo arrangements were unbalanced. The MILP model was able to find the optimal or a feasible solution in $99.9 \%$ of the unbalanced solutions, which means that the RH was only used twice. In average, 14.1 boxes were rearranged in this subset of instances (cargo of customers 2 and 3). The MILP model was able to obtain better results in this scenario, mainly because the average occupation volume decreased from $93.9 \%$ to $76.3 \%$, therefore increasing the flexibility of the boxes' movement.

Finally, Table 4 presents the results concerning the instances with just the cargo of the last customer to be visited. Only three cargo arrangements were naturally balanced. The MILP model was able to find the optimal solution in $97 \%$ of the unbalanced cargo arrangements and the RH was only used in three cargo arrangements where the MILP was not able to obtain a feasible solution in $600 \mathrm{~s}$. 
Table 5

Computational results for the MLBRA with the multi-drop container loading algorithm.

\begin{tabular}{|c|c|c|c|c|c|c|c|c|}
\hline \multirow[b]{2}{*}{ Instance } & \multicolumn{4}{|c|}{ Maximum Volume Occupation } & \multicolumn{4}{|c|}{ Minimum Box Movement } \\
\hline & Route & $\begin{array}{l}\text { Volume } \\
(\%)\end{array}$ & $\begin{array}{l}\text { \#Boxes not } \\
\text { delivered }\end{array}$ & $\begin{array}{l}\text { \#Boxes } \\
\text { moved }\end{array}$ & Route & $\begin{array}{l}\text { Volume } \\
\text { (\%) }\end{array}$ & $\begin{array}{l}\text { \#Boxes not } \\
\text { delivered }\end{array}$ & $\begin{array}{l}\text { \#Boxes } \\
\text { moved }\end{array}$ \\
\hline 1 & $3-4-2-1$ & 79.9 & 16 & 2 & $2-1-3-4$ & 77.4 & 19 & 0 \\
\hline 2 & $3-1-2-4$ & 83.9 & 20 & 6 & $3-2-1-4$ & 76.5 & 20 & 1 \\
\hline 3 & $3-2-1-4$ & 75.3 & 29 & 2 & $3-1-2-4$ & 71.3 & 37 & 0 \\
\hline 4 & $1-2-3-4$ & 78.1 & 30 & 4 & $4-1-2-3$ & 68.3 & 49 & 0 \\
\hline 5 & $1-2-3-4$ & 78.0 & 19 & 6 & $4-1-3-2$ & 68.3 & 40 & 0 \\
\hline 6 & $2-1-3-4$ & 84.0 & 21 & 5 & $2-4-3-1$ & 76.1 & 25 & 0 \\
\hline 7 & $1-3-2-4$ & 75.9 & 23 & 6 & $1-3-4-2$ & 71.5 & 38 & 2 \\
\hline 8 & $3-1-2-4$ & 73.4 & 23 & 36 & $1-4-2-3$ & 64.3 & 34 & 1 \\
\hline 9 & $2-1-4-3$ & 68.7 & 36 & 1 & $1-3-2-4$ & 67.2 & 36 & 0 \\
\hline 10 & $3-2-1-4$ & 80.8 & 37 & 4 & $2-1-3-4$ & 76.4 & 33 & 0 \\
\hline Average & & 77.8 & 25.4 & 7.2 & & 71.7 & 33.1 & 0.4 \\
\hline
\end{tabular}

It is worth noting that the results of the computational experiments have shown that the cargo arrangements generated by the BRKGA algorithm do not naturally comply with the load balance requirements, even for the partial cargo arrangements.

\section{Integrating the multi-drop load balance recovery algorithm with a multi-drop container loading algorithm}

This section presents an example of integration of the MDLBRA with a multi-drop container loading algorithm, considering different routes. The goal is to demonstrate the impact of the MDLBRA in container loading solutions with multi-drop constraints.

The test problems used were based on the first ten instances of the BR2 data set. However, the multi-drop container loading algorithm requires each box to be assigned to a customer. In these computational experiments, four customers were considered. The boxes were divided among the customers using a 40/30/20/10 ratio.

To solve the multi-drop container loading problem (in this set of experiments, the a priori provision of cargo configurations is not considered) the algorithm proposed by Correia et al. (1992) was used. This algorithm is based on the wallbuilding heuristic by George and Robinson (1980). Because the multi-drop container loading algorithm requires a customer sequence, the full enumeration of customer sequences generates 24 container loading problems for each one of the 10 BR2 instances, in a total of 240 instances that were run in these computational experiments.

Table 5 presents the results. The computational results were divided into two parts. On the left side of the table, the route is selected so that the volume utilisation is maximised. On the right side of the table, the route is selected so that the number of boxes moved is minimised. Both present the route corresponding to the best solution, the percentage of volume occupied when the vehicle leaves the depot, the number of boxes not delivered due to load balance concerns, and the number of boxes that were moved to a new position during the trip.

The average computational time of the MDLBRA was $64.6 \mathrm{~s}$. For all 240 instances tested, the MILP model was able to find the optimal or a feasible solution without removing boxes and the RH was never used. This can be explained by the relatively low (71.3\%) average volume occupation of the solutions obtained with the multi-drop container loading algorithm.

Analysing the results, one can conclude that the solutions selected with the goal of maximising volume occupation have a higher number of boxes moved during the journey, which was expectable. Depending on the particular logistics costs in each real-world application, the decision-maker may prefer to minimise the number of boxes not delivered or the number of boxes rearranged during the journey.

\section{Conclusions}

This paper addressed the until now untackled problem of load balancing cargo arrangements under multi-drop constraints throughout a complete distribution trip.

The solution strategy builds on a MILP model that, given a cargo arrangement, ensures load balance through the rearrangement of the cargo by moving and/or removing boxes, while respecting LDD constraints. The solution of this model is embedded in a multi-drop load balance recovery algorithm (MDLBRA) that ensures cargo load balance throughout the entire route.

The extensive computational experiments have shown that the MILP model can be used in practical contexts, since it was able to find a solution in less than ten minutes for $93 \%$ of the unbalanced cargo arrangements tested.

Finally, the MDLBRA proved its efficiency and versatility when integrated with a multi-drop container loading algorithm.

Future research on this problem will certainly consider the full integration of the container loading problem with the vehicle routing problem, taking into account cargo balance and stability constraints. This integration will simultaneously 
consider the constraints of both problems and a cost component in the objective function to account for box movement in each client.

\section{Acknowledgements}

The first author was supported by FCT - Fundação para a Ciência e a Tecnologia (Portuguese Foundation for Science and Technology) within the grant SFRH/BPD/98981/2013. The research was also supported by Project ERDF - European Regional Development Fund through the Operational Programme for Competitiveness and Internationalisation - COMPETE 2020 Programme within project "POCI-01-0145-FEDER-006961", and by National Funds through the Portuguese funding agency, FCT Fundação para a Ciência e a Tecnologia as part of project "UID/EEA/50014/2013"and project “POCI-01-0145-FEDER-029609".

\section{Supplementary material}

Supplementary material associated with this article can be found, in the online version, at 10.1016/j.trb.2018.08.001.

\section{References}

Alonso, M., Alvarez-Valdes, R., Iori, M., Parreño, F., Tamarit, J., 2017. Mathematical models for multicontainer loading problems. Omega 66, $106-117$.

Alonso, M.T., Alvarez-Valdes, R., Parreño, F., Tamarit, J.M., 2016. Algorithms for pallet building and truck loading in an interdepot transportation problem. Math. Probl. Eng. 2016.

Baldi, M.M., Perboli, G., Tadei, R., 2012. The three-dimensional knapsack problem with balancing constraints. Appl. Math. Comput. 218 (19), 9802-9818. doi:10.1016/j.amc.2012.03.052.

Bischoff, E.E., Ratcliff, M.S.W., 1995. Issues in the development of approaches to container loading. Omega 23 (4), 377-390. doi:10.1016/0305-0483(95) 00015-G.

Bortfeldt, A., Gehring, H., 2001. A hybrid genetic algorithm for the container loading problem. Eur. J. Oper. Res. 131 (1), 143-161. doi:10.1016/S0377-2217(00) 00055-2.

Bortfeldt, A., Wäscher, G., 2013. Constraints in container loading-a state-of-the-art review. Eur. J. Oper. Res. 229 (1), 1-20. doi:10.1016/j.ejor.2012.12.006.

Chan, F.T., Bhagwat, R., Kumar, N., Tiwari, M., Lam, P., 2006. Development of a decision support system for air-cargo pallets loading problem: a case study. Expert Syst. Appl. 31 (3), 472-485. doi:10.1016/j.eswa.2005.09.057.

Chen, C., Lee, S., Shen, Q., 1995. An analytical model for the container loading problem. Eur. J. Oper. Res. 80 (1), 68-76. doi:10.1016/0377-2217(94)00002-T.

Correia, M., Gomes, A., Oliveira, J., Herreira, J., 1992. Problemas de empacotamento tridimensional. Investigação operacional 12 (2), $169-180$.

Costa, M.d.G., Captivo, M.E., 2016. Weight distribution in container loading: a case study. Int. Trans. Oper. Res. 23 (1-2), 239-263. doi:10.1111/itor.12145.

Davies, A., Bischoff, E.E., 1999. Weight distribution considerations in container loading. Eur. J. Oper. Res. 114 (3), 509-527. doi:10.1016/S0377-2217(98) 00139-8.

Egeblad, J., Garavelli, C., Lisi, S., Pisinger, D., 2010. Heuristics for container loading of furniture. Eur. J. Oper. Res. 200 (3), 881-892. doi:10.1016/j.ejor.2009. 01.048.

Egeblad, J., Pisinger, D., 2009. Heuristic approaches for the two- and three-dimensional knapsack packing problem. Comput. Oper. Res. 36 (4), $1026-1049$. doi:10.1016/j.cor.2007.12.004.

Eurostat, 2017. Energy, Transport and Environment Indicators-2013 edition. Publications Office of the European Union, Luxembourg.

Fasano, G., 2004. A MIP approach for some practical packing problems: balancing constraints and tetris-like items. Q. J. Belgian FrenchItalian Oper. Res. Soc. 2 (2), 161-174

Gehring, H., Bortfeldt, A., 1997. A genetic algorithm for solving the container loading problem. Int. Trans. Oper. Res. 4 (5-6), $401-418$.

Gehring, H., Menschner, K., Meyer, M., 1990. A computer-based heuristic for packing pooled shipment containers. Eur. J. Oper. Res. 44 (2), 277-288.

George, J., Robinson, D., 1980. A heuristic for packing boxes into a container. Comput. Oper. Res. 7 (3), 147-156. doi:10.1016/0305-0548(80)90001-5.

Haessler, R.W., Brian Talbot, F., 1990. Load planning for shipments of low density products. Eur. J. Oper. Res. 44 (2), 289-299. doi:10.1016/0377-2217(90) 90364-H

Iori, M., Martello, S., 2010. Routing problems with loading constraints. TOP 18 (1), 4-27. doi:10.1007/s11750-010-0144-X.

Lim, A., Ma, H., Qiu, C., Zhu, W., 2013. The single container loading problem with axle weight constraints. Int. J. Prod. Econ. 144 (1), 358-369.

Liu, F.-H.F., Hsiao, C.-J., 1997. A three-dimensional pallet loading method for single-size boxes. J. Oper. Res. Soc. 48 (7), 726. doi:10.2307/3010061.

Liu, W.-Y., Lin, C.-C., Yu, C.-S., 2011. On the three-dimensional container packing problem under home delivery service. Asia-Pacific J. Oper. Res. 28 (05), 601-621. doi:10.1142/S0217595911003466.

Moon, I., Nguyen, T.V.L., 2014. Container packing problem with balance constraints. OR Spectrum 36 (4), $837-878$.

Øvstebø, B.O., Hvattum, L.M., Fagerholt, K., 2011. Routing and scheduling of RoRo ships with stowage constraints. Transp. Res. Part C 19 (6), 1225-1242.

Paquay, C., Limbourg, S., Schyns, M., 2018. A tailored two-phase constructive heuristic for the three-dimensional multiple bin size bin packing problem with transportation constraints. Eur. J. Oper. Res. 267 (1), 52-64.

Pollaris, H., Braekers, K., Caris, A., Janssens, G.K., Limbourg, S., 2015. Vehicle routing problems with loading constraints: state-of-the-art and future directions. OR Spectrum 37 (2), 297-330.

Pollaris, H., Braekers, K., Caris, A., Janssens, G.K., Limbourg, S., 2016. Capacitated vehicle routing problem with sequence-based pallet loading and axle weight constraints. EURO J. Transp. Logist. 5 (2), 231-255.

Pollaris, H., Braekers, K., Caris, A., Janssens, G.K., Limbourg, S., 2017. Iterated local search for the capacitated vehicle routing problem with sequence-based pallet loading and axle weight constraints. Networks 69 (3), 304-316.

Ramos, A.G., Oliveira, J.F., Gonçalves, J.F., Lopes, M.P., 2016. A container loading algorithm with static mechanical equilibrium stability constraints. Transp. Res. Part B 91, 565-581

Ramos, A.G., Silva, E., Oliveira, J.F., 2018. A new load balance methodology for container loading problem in road transportation. Eur. J. Oper. Res. 266 (3), $1140-1152$.

Techanitisawad, A., Tangwiwatwong, P., 2004. A GA-based heuristic for the interrelated container selection loading problems. IEMS 3 (1), 22-37.

Tricoire, F., Parragh, S.N., 2017. Investing in logistics facilities today to reduce routing emissions tomorrow. Transp. Res. Part B 103 , 56-67.

Volvo Trucks, 2015. Data sheet specification model range FE 62TR. Retrieved January 12, 2016, from http://segotn12827.rds.volvo.com/stpifiles/volvo/ modelrange/fe62tra_aus_engau.pdf.

Wang, L., Guo, S., Chen, S., Zhu, W., Lim, A., 2010. Two natural heuristics for 3D packing with practical loading constraints. In: PRICAI 2010: Trends in Artificial Intelligence. Springer, pp. 256-267.

Zachariadis, E.E., Tarantilis, C.D., Kiranoudis, C.T., 2015. The load-dependent vehicle routing problem and its pick-up and delivery extension. Transp. Res. Part B 71, 158-181. 